\title{
Tooth discoloration induced by calcium-silicate-based pulp-capping materials
}

\author{
Da-A Yun ${ }^{1}$, Su-Jung Park ${ }^{1}$, Seok-Ryun Lee ${ }^{1}$, Kyung-San Min²,3
}

Correspondence: Dr. Kyung-San Min

Email: endomin@gmail.com

\begin{abstract}
'Department of Conservative Dentistry, School of Dentistry, Wonkwang University, Iksan, Korea, 2Department of Conservative Dentistry, School of Dentistry and Institute of Oral Bioscience, Chonbuk National University, Jeonju, Korea,

${ }^{3}$ Research Institute of Clinical Medicine of Chonbuk National University-Biomedical Research Institute of Chonbuk National University Hospital, Jeonju, Korea
\end{abstract}

\section{ABSTRACT}

Objectives: The aim of this study was to evaluate tooth discoloration induced by contact with various calcium silicate-based pulp capping materials in the presence or absence of blood in vitro. Materials and Methods: Eighty bovine samples were divided into six experimental groups and two control groups according to the type of material used (ProRoot [PR], Endocem [EC], or EndocemZr [ECZ]) and the presence or absence of contamination with blood. A spectrophotometer was used to calculate the color difference $(\Delta \mathrm{E})$ between the baseline measurement (after placement of materials) and measurements taken $1,2,4$, and 8 weeks. The results were analyzed with repeated measures analysis of variance, Tukey's post-hoc tests and independent $t$-tests $(P=0.05)$. Results: The PR group and EC group showed significantly higher mean values of $\Delta \mathrm{E}$ than the negative control group after 2 weeks $(P<0.05)$, whereas $\mathrm{ECZ}$ did not. There were larger $\Delta \mathrm{E}$ values when there was contact with blood, especially in PR and EC group $(P<0.05)$. Conclusions: ECZ which contains zirconium oxide as a radiopacifier showed less discoloration irrespective of blood contamination compared to PR and EC.

Key words: Calcium silicate, pulp capping, radiopacifier, tooth discoloration, zirconium

\section{INTRODUCTION}

Mineral trioxide aggregate (MTA) is a useful material for vital pulp therapy, such as pulp capping and pulpotomy..$^{[1-5]}$ In these clinical situations, MTA is used in a coronal position; therefore, the color of MTA is considered key to the final esthetic result. ${ }^{[6]}$ In the 1990s, Torabinejad et al. introduced gray MTA (GMTA), which has a similar chemical constitution to that of Portland cement except for the addition of bismuth oxide $\left(\mathrm{Bi}_{2} \mathrm{O}_{3}\right)(17-18 \mathrm{wt} \%)$ to improve radioopacity. ${ }^{[7,8]}$ Despite its favorable biological features, GMTA causes tooth discoloration as well as discoloration of the adjacent gingiva. ${ }^{[4,6]}$ Because of this aesthetic problem, white MTA (WMTA) was developed through the exclusion of iron compounds rather than by the addition of other elements (ProRoot (PR); Dentsply, Tulsa, OK, USA). ${ }^{[9]}$ However, there have been many reports of tooth discoloration with PR. ${ }^{[10-12]}$ Recently, Belobrov and Parashos reported a case of tooth discoloration after use of WMTA as a pulp-capping material. ${ }^{[13]}$

In response to these issues, a pozzolan-based MTA-derived material (Endocem(EC); Maruchi, Wonju, Korea) was introduced into the endodontic market. The advantages of EC are its reduced setting time and improved washout resistance. ${ }^{[14,15]}$ Furthermore, the biocompatibility and osteogenicity/odontogenicity of EC are similar to those of PR. ${ }^{[15,16]}$ However, there is still the possibility of tooth discoloration when using this material. Recent studies of color stability of

\footnotetext{
How to cite this article: Yun DA, Park SJ, Lee SR, Min KS. Tooth discoloration induced by calcium-silicate-based pulp-capping materials. Eur J Dent 2015;9:165-70.

Copyright $\odot 2015$ Dental Investigations Society.

DOI: $10.4103 / 1305-7456.156789$
} 
WMTA have suggested that the formation of metallic bismuth under light irradiation can lead to color change. ${ }^{[17,18]}$ Thus, the use of an alternative radiopacifier to replace bismuth in WMTA is needed. ${ }^{[19]}$ Recently, the manufacturer that produces EC released another fast-setting pozzolan cement that contains zirconium oxide $\left(\mathrm{ZrO}_{2}\right)$ as a radiopacifier (EndocemZr [ECZ]; Maruchi) to overcome the gray color of original EC. It has been reported that $\mathrm{ZrO}_{2}$ has acceptable radiopacity ${ }^{[20]}$ and does not participate in the hydration reaction of Portland cement. ${ }^{[21]}$ Recently, the addition of $30 \% \mathrm{ZrO}_{2}$ to PC exhibited physical and antimicrobial properties comparable to those of PC. ${ }^{[22,23]}$ However, there is little information about the effect of ECZ on tooth discoloration.

In various clinical situations, such as pulp capping and perforation repair, MTA or MTA-derived materials are often applied in contact with blood. It is generally believed that blood can cause tooth discoloration. ${ }^{[24,25]}$ Furthermore, even blood in the root canal adjacent to the WMTA has been shown to influence discoloration. ${ }^{[26]}$ Therefore, the aim of this study was to evaluate tooth discoloration induced by contact with various calcium silicate-based pulp-capping materials in the presence or absence of blood in vitro.

\section{MATERIALS AND METHODS}

\section{Tooth preparation}

A total of 80 intact bovine incisors were used in this study. Exclusion criteria were the presence of caries, visible structural defects, coronal staining, and narrow crown width (labial surface area $>10 \times 10 \mathrm{~mm}^{2}$ ). The samples were formed with reference to a new model introduced by a previous study, as shown in Figure 1. ${ }^{[27]}$ Briefly, the teeth were disinfected in 1\% chloramine-T solution (Sigma-Aldrich, St. Louis, MO, USA) and stored in normal saline at room temperature for 1-month. The

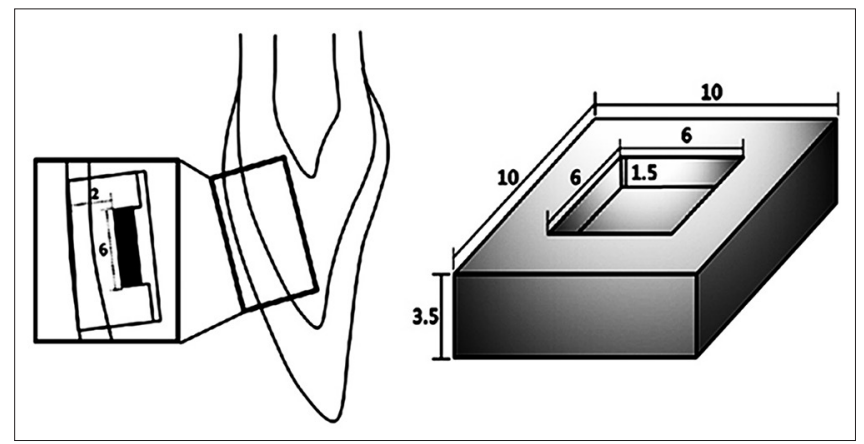

Figure 1: A standardized cuboid enamel-dentin block $(10 \mathrm{~mm} \times 10 \mathrm{~mm} \times 3.5 \mathrm{~mm})$, which was prepared by removing the middle third of a bovine incisor roots were resected using a diamond-coated disc, and extrinsic stains and calculus on the labial surface were removed with an ultrasonic scaler. A cuboid enamel-dentin block $(10 \mathrm{~mm} \times 10 \mathrm{~mm} \times 3.5 \mathrm{~mm})$ was prepared from the middle third of each crown using a low-speed microtome (ISOMET, Buehler, IL, USA). The labial enamel surface was finished and polished with 220, 600, 1200, and 2000-grit abrasive papers (CC261; DEERFOS, Seoul, Korea). The height of each block was standardized at $3.5 \mathrm{~mm} \pm 0.1 \mathrm{~mm}$. Then, a box-form cavity $(6 \mathrm{~mm} \times 6 \mathrm{~mm} \times 1.5 \mathrm{~mm})$ was prepared with a \#330 carbide bur in the middle of each specimen, leaving $2 \mathrm{~mm}$ of the labial tooth structure $(1 \mathrm{~mm}$ each of enamel and dentin). Sodium hypochlorite $(1.5 \% \mathrm{NaOCl})$ irrigation solution was placed in the cavity for $30 \mathrm{~min}$, and $17 \%$ ethylene diamine tetraacetic acid (EDTA; PrevestDentpro, Jammu, India) was applied for $2 \mathrm{~min}$ to remove the smear layer and to expose the dentinal tubules. Cavities were irrigated with a final rinse of $1 \% \mathrm{NaOCl}$ and saline. All specimens were stored in a container at $100 \%$ relative humidity.

\section{Blood collection}

Human blood was used to simulate the clinical situation of vital pulp therapy. In this study, whole blood was collected from a volunteer (experimenter). The blood collection tubes (Vacuette®; Greiner Bio-One, Kremsmünster, Austria) were sterile and spray-coated with the anticoagulant $\mathrm{K}_{2}$ EDTA to prevent clotting.

\section{Sample preparation for experimental and control groups}

The three materials included in this study were PR, EC, and ECZ [Table 1]. The specimens were randomly divided into six experimental groups and two control groups according to the material used and the presence or absence of contamination with blood [Table 2]. Each material was mixed according to the manufacturer's instructions and was placed into the cavity. In the blood-contaminated groups, $5 \mu \mathrm{L}$ of blood was placed on top of the material. A negative control (NC) was filled with a cotton pellet moistened with normal saline, and a positive control was filled with a cotton

\section{Table 1: Composition of tested materials}

\begin{tabular}{ll}
\hline Material & Composition \\
\hline PR & $\begin{array}{l}\text { Tricalcium silicate, dicalcium silicate, tricalcium } \\
\text { aluminate, tetracalcium aluminoferrite, } \\
\text { free calcium oxide, bismuth oxide }\end{array}$ \\
EC & $\begin{array}{l}\text { Calcium oxide, silicate oxide, aluminum } \\
\text { oxide, other metallic oxides, bismuth oxide } \\
\text { Calcium oxide, silicate oxide, aluminum oxide, } \\
\text { other metallic oxides, zirconium oxide }\end{array}$ \\
\hline
\end{tabular}

PR: ProRoot, EC: Endocem, ECZ: EndocemZr 


\begin{tabular}{ll} 
Table 2: Teeth and materials in experimental and \\
control groups \\
\hline Group & Material \\
\hline Blood-free condition & \\
PR & PR+saline \\
EC & EC+saline \\
ECZ & ECZ+saline \\
NC & NC (cotton+saline) \\
Blood-contaminated condition & \\
PR/B & PR+blood \\
EC/B & EC+blood \\
ECZ/B & ECZ+blood \\
PC & PC (cotton+blood) \\
\hline PR: ProRoot, EC: Endocem, ECZ: EndocemZr, PC: Positive control, \\
NC: Negative control
\end{tabular}

pellet moistened with blood. Then, the cavities were sealed with a self-adhesive resin material (U-Cem Universal; Vericom, Chuncheon, Korea). An LED curing light (Elipar ${ }^{\mathrm{TM}}$ S10; 3M ESPE, Munich, Germany) was used to irradiate the specimens for $20 \mathrm{~s}$. All specimens were stored at room temperature and $100 \%$ relative humidity.

\section{Evaluation of tooth discoloration}

As shown in Figure 2, a standardized acrylic resin mold was developed to allow measurement of the same position in each sample and to increase reproducibility. The sample was positioned in the standardized mold and adjusted to a reference line. The tooth color was recorded at baseline $\left(\mathrm{D}_{0}\right.$; after tooth preparation and placement of materials) and at 1-week $\left(D_{7}\right)$, 2 weeks $\left(D_{14}\right), 4$ weeks $\left(D_{28}\right)$, and 8 weeks $\left(D_{56}\right)$ using a spectrophotometer (NF999; Nippon Denshoku, Tokyo, Japan). All measurements were repeated three times and averaged. The color differences $(\triangle \mathrm{E})$ between the baseline and the $\mathrm{D}_{7}, \mathrm{D}_{14^{\prime}} \mathrm{D}_{28^{\prime}} \mathrm{D}_{56}$ measurements were calculated using the following formula: $\Delta \mathrm{E}=\left(\left[\mathrm{L}{ }_{2}{ }_{2}-\mathrm{L} *{ }_{1}\right]^{2}+\left[\mathrm{a}{ }^{*}{ }_{2}-\mathrm{a}{ }^{*}{ }_{1}\right]^{2}+\left[\mathrm{b}{ }^{*}{ }_{2}-\mathrm{b}{ }^{*}{ }_{1}\right]^{2}\right]^{1 / 2}$. The value of the luminosity $\left(\mathrm{L}^{*}\right)$ represents the lightness values, $a^{*}$ represents the values of red-green, and $b^{*}$ represents the values of yellow-blue.

\section{Stereomicroscopic observation of representative samples}

In each group, a representative sample was selected and sectioned in the center by using a low-speed microtome (ISOMET). The sectioned surface was examined under a stereomicroscope (Leica MZ16FA; Leica, Wezler, Germany).

\section{Statistical analysis}

The data were evaluated using SPSS Software (PASW Statistics 20; SPSS Inc., Chicago, IL, USA). Statistical

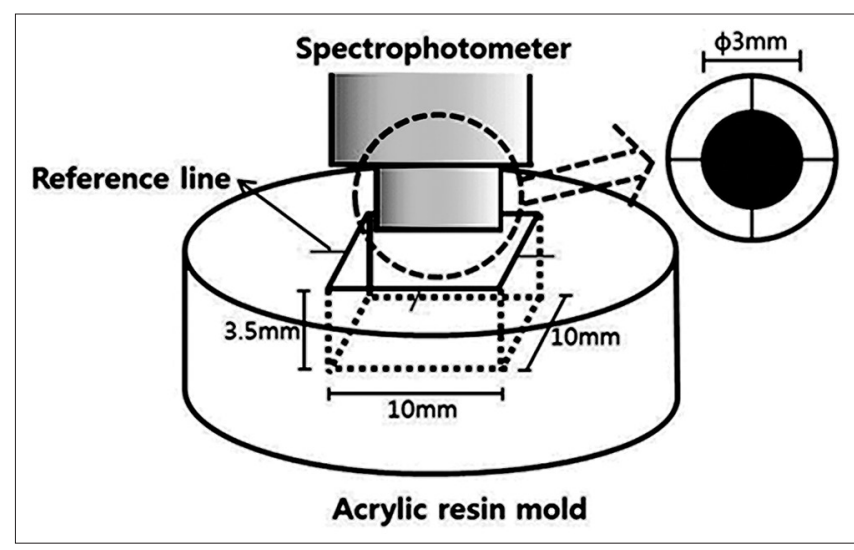

Figure 2: Standardized acrylic resin mold used to repeatedly measure the same position in each sample

analysis was performed using repeated measures analysis of variance to examine the $\Delta \mathrm{E}$ values as the dependent variables and time as a factor. Tukey's post hoc test was used to assess significant differences between the tested materials. Independent $t$-test was applied to determine the influence of blood contamination. A $P<0.05$ was considered statistically significant for the tests.

\section{RESULTS}

Color differences $(\triangle E)$ of teeth in contact with the tested materials

The mean, standard deviation, and significance of the color differences are shown in Figure 3. When the $\Delta \mathrm{E}$ value is higher than 3.3 , it is possible to distinguish the color difference of the tooth. ${ }^{[28]}$ After 1 week, the $\triangle \mathrm{E}$ value was higher than 3.3 in all experimental groups. We observed that all teeth experienced color alteration after contact with the tested materials. The PR group and EC group showed significantly higher mean values than the NC group after 2 weeks and 1 week, respectively $(P<0.05)$. On the contrary, in the ECZ group, the color of the tested specimens was not significantly different from that of the $\mathrm{NC}$, with the exception of data at $\mathrm{D}_{7}(P>0.05)$.

Color differences $(\Delta \mathrm{E})$ of teeth in contact with the materials and blood

There were larger color differences ( $\triangle \mathrm{E}$ value) when in contact with blood irrespective of time or type of material [Figure 4]. In comparison with the positive control, the color changes of all experimental groups were not significantly different at most time points $(P>0.05)$. After 2 weeks, the $P R / B$ group showed a higher $\triangle \mathrm{E}$ value than the $\mathrm{ECZ} / \mathrm{B}$ group; however, a significant difference was only observed at $\mathrm{D}_{56}(P<0.05)$. Furthermore, the EC/B group $(\mathrm{G} 7)$ 


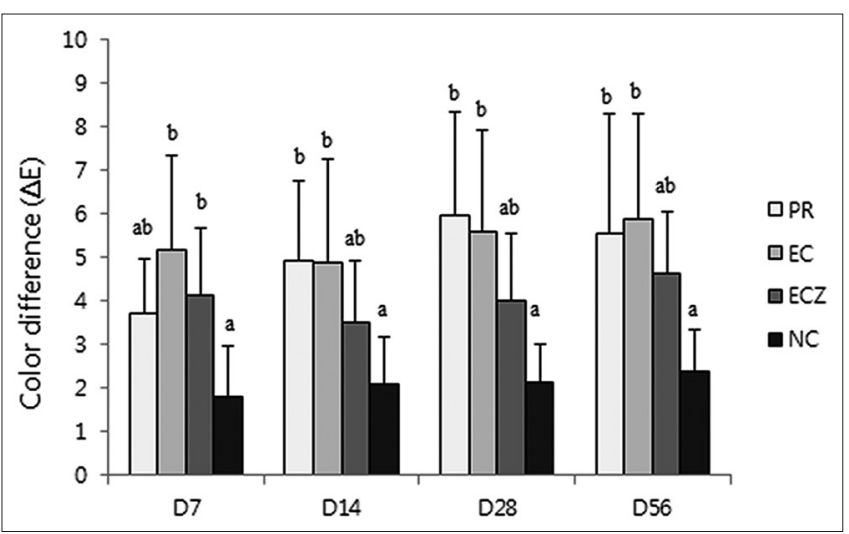

Figure 3: $\Delta \mathrm{E}$ Values for the different groups at the different time points (group 1-4). The values of $\Delta \mathrm{E}>3.3$ are considered appreciable by non-skilled persons. The same letters indicate statistically similar groups $(P=0.05)$

showed a larger color difference than the ECZ/B group, but the difference was only significant at $\mathrm{D}_{28}(P<0.05)$.

\section{DISCUSSION}

Discoloration of anterior teeth is an esthetic concern. Several case reports have revealed discoloration produced by MTA when the material was used as a pulp-capping or pulpotomy agent. . $^{[4,13,29]}$ However, few studies have evaluated the discoloration of various MTA-derived materials, especially $\mathrm{ZrO}_{2}$-containing materials. In the present study, the $\Delta \mathrm{E}$ value was higher than 3.3 in all experimental groups after 1-week [Figure 3]. We observed all teeth to have experienced color alteration after contact with MTA materials, which is consistent with findings of previous studies. ${ }^{[26,27]}$ The present study showed that the PR and EC groups experienced significantly more discoloration than the NC group after 2 weeks. However, there were no significant color changes between the ECZ group and the NC. A possible explanation for these results is that the $\mathrm{Bi}_{2} \mathrm{O}_{3}$ included in PR and EC causes discoloration. One possible mechanism of this change is that the $\mathrm{Bi}_{2} \mathrm{O}_{3}$ interacts with collagen in dentin and is converted to a black precipitate in the material-dentin interface. A recent investigation showed that collagen, which is present in organic dentin matrix, reacts with $\mathrm{Bi}_{2} \mathrm{O}_{3}$, resulting in grayish discoloration. ${ }^{[19]}$ Another hypothesis to explain the discoloration is the formation of metallic bismuth under specific conditions. Irradiated $\mathrm{Bi}_{2} \mathrm{O}_{3}$ under oxygen-free conditions is excited and changed to dark grayish metallic bismuth. ${ }^{[17]} \mathrm{A}$ recent study also reported that contact of WMTA and other bismuth-containing materials with $\mathrm{NaOCl}$ produces a change to a darker color because the oxide is converted to bismuth metal in contact with sodium hypochlorite and oxygen is lost. ${ }^{[30]}$

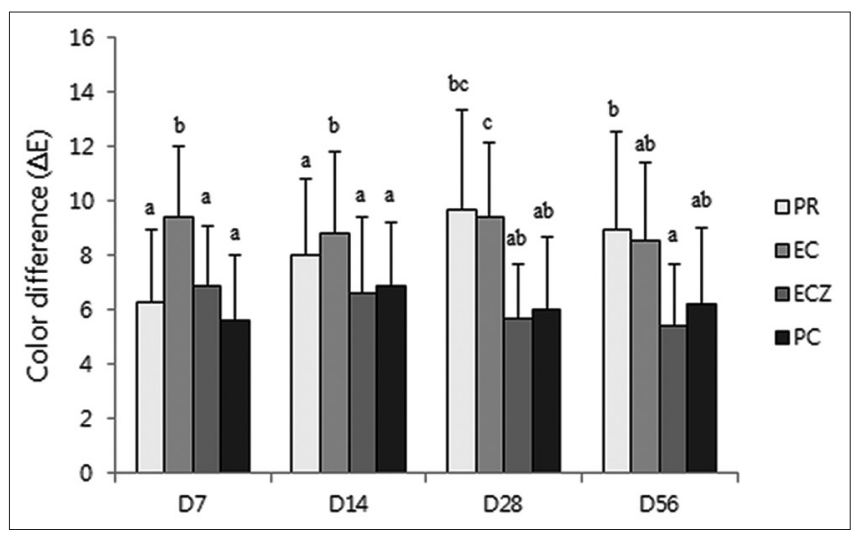

Figure 4: $\Delta \mathrm{E}$ Values for the different blood contaminated groups at the different time points (group 5-8). The values of $\Delta \mathrm{E}>3.3$ are considered appreciable by non-skilled persons. The same letters indicate statistically similar groups $(P=0.05)$

Our stereomicroscopic examination of the PR group revealed grayish discoloration in the interfacial dentin and the material itself [Figure 5a]. However, the color of the EC changed the color of the specimen to dark gray [Figure 5b]. A recent in vitro study supported our results that significant discoloration occurs in the cervical area in the presence of PR, MTA Angelus, and EC. ${ }^{[31]}$ One possibility is that the organic matrix in the dentin surface reacts with $\mathrm{Bi}_{2} \mathrm{O}_{3^{\prime}}$ a possible major factor contributing to discoloration, and the remaining $\mathrm{NaOCl}$ solution in the cavities of the specimens might exacerbate the change in color through reaction with the $\mathrm{Bi}_{2} \mathrm{O}_{3}$ included in the PR and EC. On the other hand, in the present study, the color of the ECZ group remained relatively stable according to spectrophotometric [Figure 3] and stereomicroscopic analyses [Figure 5c]. These results might be explained by the inclusion of $\mathrm{ZrO}_{2}$ as a radiopacifier instead of $\mathrm{Bi}_{2} \mathrm{O}_{3}$, as well as the light color of the material itself. In other words, aside from the original dark color of the material, calcium silicate-based materials containing $\mathrm{Bi}_{2} \mathrm{O}_{3}$ undergo discoloration under conditions that simultaneously include contact with an organic dentin matrix, an oxygen-free environment, and irradiation with a curing light. Therefore, when selecting pulp-capping materials for areas of aesthetic concern, ECZ, which includes $\mathrm{ZrO}_{2}$ rather than $\mathrm{Bi}_{2} \mathrm{O}_{3}$, might be a better option.

The sequential color change $(\Delta \mathrm{E})$ of teeth in contact with materials and blood is presented in Figure 4. The specimens showed an increased $\Delta \mathrm{E}$ value after contamination with blood at each time point. However, a significant difference was observed in the PR/B and EC/B groups only after 8 weeks. On stereomicroscopic examination, the PR/B 


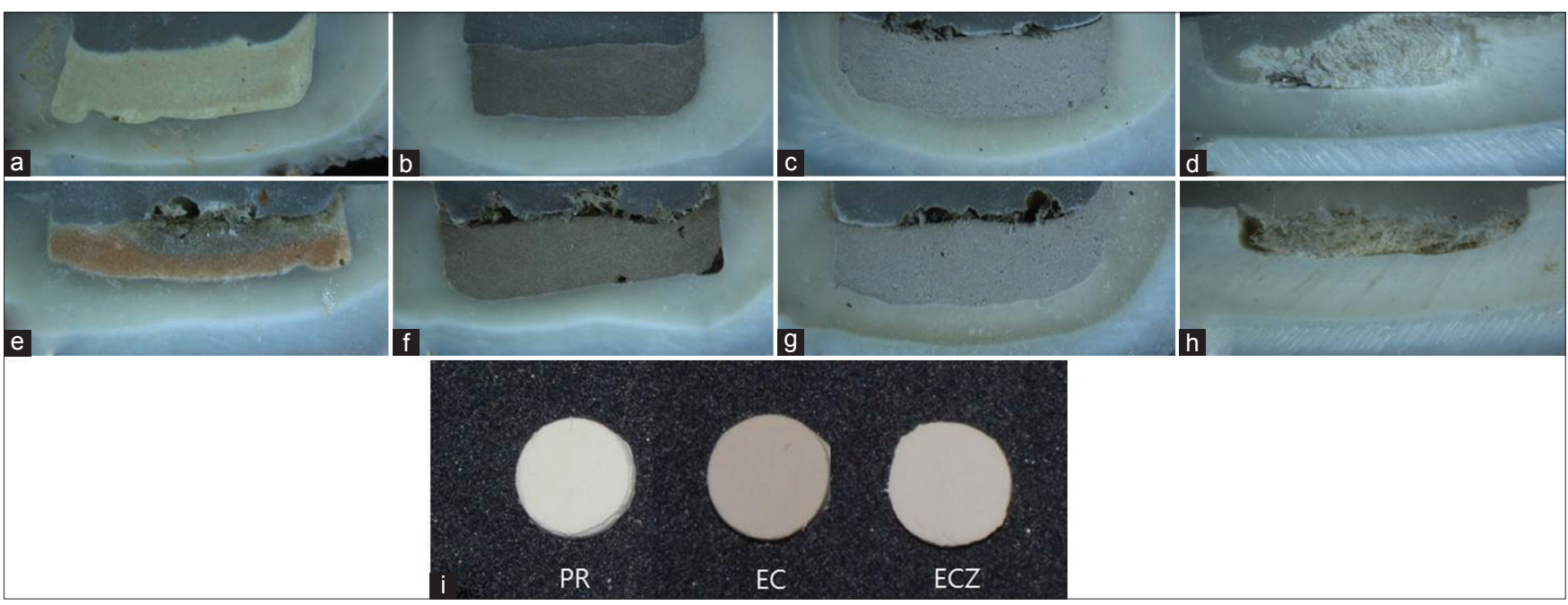

Figure 5: Stereomicroscopic appearance of a representative sample from each group after 8 weeks; (a) PR, (b) EC, (c) ECZ, (d) negative control, (e) ProRoot with blood, (f) EC with blood, (g) ECZ with blood, and (h) positive control. (i) Samples show the initial color of each material; PR: ProRoot, EC: Endocem, ECZ: EndocemZr

specimen [Figure 5e] showed greater discoloration of the material itself, demonstrating a dark orange to gray color change. This result is in agreement with the findings of a previous study that reported greater discoloration of the WMTA/blood group compared to the WMTA/saline group. ${ }^{[26]}$ Therefore, we conclude that the presence of blood within the cavity exacerbates tooth discoloration induced by MTA-based pulp capping materials. One possible mechanism may be the interaction between erythrocytes in the blood and the unset MTA-based pulp-capping materials. Incompletely set materials may allow absorption of blood and hemolysis of erythrocytes from the vital pulp tissue. ${ }^{[26]}$ However, EC is known as a fast setting material, eliminating the possibility of this explanation here. Another hypothesis is that the material includes $\mathrm{Bi}_{2} \mathrm{O}_{3}$ as a radiopacifier, which might result in progressive mass darkening due to interaction with the organic matrix in blood. However, after 8 weeks, the discoloration value of the ECZ group was not significant regardless of the presence or absence of blood. We speculated that, because ECZ is a fast setting material, it does not allow for absorption of blood, and it does not include $\mathrm{Bi}_{2} \mathrm{O}_{3}$, which might explain the absence of progressive discoloration of the specimens. Consequently, the PR/B and EC/B groups showed greater discoloration compared with the ECZ group, and contamination with blood adversely affected the color stability of the tooth. However, the exact mechanism remains unknown, indicating the need for additional research.

In this study, bovine incisors were used to evaluate tooth discoloration because they have many advantages compared with human teeth. First, a sufficient number of intact incisors can be obtained. Second, the number of tubules per $\mathrm{mm}^{2}$ and the tubule diameter of bovine incisors are not significantly different than those of the coronal dentin of human teeth. ${ }^{[32]}$ Furthermore, bovine incisors have wide and flat surfaces; therefore, a standardized sample can be easily obtained. Although bovine incisors have been widely used as specimens for in vitro studies of discoloration, there is a need for additional research using human incisors.

\section{CONCLUSION}

Within the limitations of the present study, we concluded that the largest color alteration was observed in the presence of PR and EC compared to ECZ. Furthermore, the presence of blood within the cavity adjacent to the materials exacerbated tooth discoloration induced by MTA-based pulp-capping materials. However, the type and compounds of the MTA-based pulp-capping material play the most significant role in the discoloration. Therefore, ECZ induces less discoloration and has the potential as an alternative pulp-dressing material for use in aesthetically sensitive areas. However, more research is required to evaluate the color stability of MTA-based materials in clinical settings.

\section{REFERENCES}

1. Iwamoto CE, Adachi E, Pameijer CH, Barnes D, Romberg EE, Jefferies S. Clinical and histological evaluation of white ProRoot MTA in direct pulp capping. Am J Dent 2006;19:85-90. 
2. Hilton TJ. Keys to clinical success with pulp capping: A review of the literature. Oper Dent 2009;34:615-25.

3. Parirokh M, Asgary S, Eghbal MJ, Stowe S, Eslami B, Eskandarizade A, et al. A comparative study of white and grey mineral trioxide aggregate as pulp capping agents in dog's teeth. Dent Traumatol 2005;21:150-4.

4. Naik S, Hegde AH. Mineral trioxide aggregate as a pulpotomy agent in primary molars: An in vivo study. J Indian Soc Pedod Prev Dent 2005;23:13-6.

5. Maroto M, Barbería E, Planells P, García Godoy F. Dentin bridge formation after mineral trioxide aggregate (MTA) pulpotomies in primary teeth. Am J Dent 2005;18:151-4.

6. Bortoluzzi EA, Araújo GS, Guerreiro Tanomaru JM, Tanomaru-Filho M. Marginal gingiva discoloration by gray MTA: A case report. J Endod 2007;33:325-7.

7. Camilleri J, Montesin FE, Brady K, Sweeney R, Curtis RV, Ford TR. The constitution of mineral trioxide aggregate. Dent Mater 2005;21:297-303.

8. Torabinejad M, Hong CU, McDonald F, Pitt Ford TR. Physical and chemical properties of a new root-end filling material. J Endod 1995;21:349-53.

9. Asgary S, Parirokh M, Eghbal MJ, Brink F. Chemical differences between white and gray mineral trioxide aggregate. J Endod 2005;31:101-3.

10. Watts JD, Holt DM, Beeson TJ, Kirkpatrick TC, Rutledge RE. Effects of $\mathrm{pH}$ and mixing agents on the temporal setting of tooth-colored and gray mineral trioxide aggregate. J Endod 2007;33:970-3.

11. Boutsioukis C, Noula G, Lambrianidis T. Ex vivo study of the efficiency of two techniques for the removal of mineral trioxide aggregate used as a root canal filling material. J Endod 2008;34:1239-42.

12. Jacobovitz M, de Lima RK. Treatment of inflammatory internal root resorption with mineral trioxide aggregate: A case report. Int Endod J 2008;41:905-12.

13. Belobrov I, Parashos P. Treatment of tooth discoloration after the use of white mineral trioxide aggregate. J Endod 2011;37:1017-20.

14. Jang GY, Park SJ, Heo SM, Yu MK, Lee KW, Min KS. Washout resistance of fast-setting pozzolan cement under various root canal irrigants. Restor Dent Endod 2013;38:248-52.

15. Choi Y, Park SJ, Lee SH, Hwang YC, Yu MK, Min KS. Biological effects and washout resistance of a newly developed fast-setting pozzolan cement. J Endod 2013;39:467-72.

16. Park SJ, Heo SM, Hong SO, Hwang YC, Lee KW, Min KS. Odontogenic effect of a fast-setting pozzolan-based pulp capping material. J Endod 2014:40:1124-31.

17. Vallés M, Mercadé M, Duran-Sindreu F, Bourdelande JL, Roig M. Influence of light and oxygen on the color stability of five calcium silicate-based materials. J Endod 2013;39:525-8.

18. Vallés M, Mercadé M, Duran-Sindreu F, Bourdelande JL, Roig M. Color stability of white mineral trioxide aggregate. Clin Oral Investig 2013;17:1155-9.

19. Marciano MA, Costa RM, Camilleri J, Mondelli RF, Guimarães BM, Duarte MA. Assessment of color stability of white mineral trioxide aggregate angelus and bismuth oxide in contact with tooth structure. J Endod 2014;40:1235-40.

20. Bortoluzzi EA, Guerreiro-Tanomaru JM, Tanomaru-Filho M, Duarte MA. Radiographic effect of different radiopacifiers on a potential retrograde filling material. Oral Surg Oral Med Oral Pathol
Oral Radiol Endod 2009;108:628-32

21. Camilleri J, Cutajar A, Mallia B. Hydration characteristics of zirconium oxide replaced Portland cement for use as a root-end filling material. Dent Mater 2011;27:845-54.

22. Weckwerth PH, Machado AC, Kuga MC, Vivan RR, Polleto Rda S, Duarte MA. Influence of radiopacifying agents on the solubility, $\mathrm{pH}$ and antimicrobial activity of portland cement. Braz Dent J 2012;23:515-20.

23. Cutajar A, Mallia B, Abela S, Camilleri J. Replacement of radiopacifier in mineral trioxide aggregate; characterization and determination of physical properties. Dent Mater 2011;27:879-91.

24. Freccia WF, Peters DD. A technique for staining extracted teeth: A research and teaching aid for bleaching. J Endod 1982;8:67-9.

25. Marin PD, Bartold PM, Heithersay GS. Tooth discoloration by blood: An in vitro histochemical study. Endod Dent Traumatol 1997;13:132-8.

26. Felman D, Parashos P. Coronal tooth discoloration and white mineral trioxide aggregate. J Endod 2013;39:484-7.

27. Lenherr P, Allgayer N, Weiger R, Filippi A, Attin T, Krastl G. Tooth discoloration induced by endodontic materials: A laboratory study. Int Endod J 2012;45:942-9.

28. Inokoshi S, Burrow MF, Kataumi M, Yamada T, Takatsu T. Opacity and color changes of tooth-colored restorative materials. Oper Dent 1996;21:73-80.

29. Karabucak B, Li D, Lim J, Iqbal M. Vital pulp therapy with mineral trioxide aggregate. Dent Traumatol 2005;21:240-3.

30. Camilleri J. Color stability of white mineral trioxide aggregate in contact with hypochlorite solution. J Endod 2014;40:436-40.

31. Jang JH, Kang M, Ahn S, Kim S, Kim W, Kim Y, et al. Tooth discoloration after the use of new pozzolan cement (Endocem) and mineral trioxide aggregate and the effects of internal bleaching. J Endod 2013;39:1598-602.

32. Schilke R, Lisson JA, Bauss O, Geurtsen W. Comparison of the number and diameter of dentinal tubules in human and bovine dentine by scanning electron microscopic investigation. Arch Oral Biol 2000;45:355-61.

\begin{tabular}{|l|l|}
\hline \multicolumn{3}{|c|}{ Access this article online } \\
\hline Quick Response Code: & $\begin{array}{l}\text { Website: } \\
\text { www.eurjent.com }\end{array}$ \\
\cline { 2 - 4 } & $\begin{array}{l}\text { Source of Support: This study was } \\
\text { supported by (1) Basic Science Research } \\
\text { Program through the National Research } \\
\text { Foundation of Korea (NRF) funded by } \\
\text { the Ministry of Education, Science and } \\
\text { Technology (2013-R1A1A4A01006389), } \\
\text { and (2) the Ministry of Trade, Industry } \\
\text { \& Energy (MOTIE), Korea Institute for } \\
\text { Advancement of Technology (KIAT), and } \\
\text { Gangwon Institute for Regional Program } \\
\text { Evaluation (GWIRPE) through the Leading } \\
\text { Industry Development for Economic Region. } \\
\text { Conflict of Interest: None declared }\end{array}$ \\
\hline
\end{tabular}

